\title{
Estructura y composición de las comunidades de palmas presentes en cinco localidades (San Juan, Tutunendo, Alto Baudó, Lloró y Raspadura) del departamento del Chocó, Colombia
}

\author{
Structure and composition of the communities of palms in five locations \\ (San Juan, Tutunendo, Alto Baudó, Lloró and Raspadura) of the department \\ of Choco, Colombia
}

\author{
Giovanny Ramírez Moreno, MSc ${ }^{1}$, Zulmary Valoyes Cardozo, Biol ${ }^{2}$, \\ Eva Ledezma Rentería, MSc ${ }^{3}$
}

\section{RESUMEN}

Desde el punto de vista de la importancia en la composición de los bosques, las palmas se encuentran entre las siete familias de plantas con mayor número de especies en los bosques bajos del Pacífico. Los muestreos relevan que en la región del Pacífico, se encuentra un mayor número de individuos de palmas porárea que en cualquier otra región del mundo. El presente estudio se basa en una serie de muestreos de palmas realizados en el marco de las expediciones biológicas de la Universidad del Chocó (2004-2005) y el estudio integral de la flora del Pacífico del Instituto de Investigaciones Ambientales del Pacífico (IIAP) (2007), con el propósito de analizar la diversidad, distribución y composición del recurso palma con alto potencial de aprovechamiento en las estaciones ambientales del IIAP en Tutunendo, Alto San Juan y Alto Baudó en Chocó. Se calculó la diversidad alfa, beta y gama; además, se utilizaron los programas estadisticos de Biodiversity Pro para hacer los análisis de diversidad alfa y la medición de los indices de Shannon $H^{\prime}$ y $J^{\prime}$, Berger Paker, Simpson, Margalet, para graficary analizar los modelos de distribución y agrupación de las especies en la comunidad; EstimateS, para el análisis de los datos y la obtención de los porcentajes de los observados y los estimadores de Chao 2, Jack 2 y Coleman y MM; y Past para obtener $X^{2}$ y el valor de P y para hacer el análisis de componentes principales (PCA). Las palmas se constituyeron en un componente dominante en la vegetación de los sitios muestreados, con una gran variedad de especies con alto potencial para usos locales. En estos muestreos se encontraron 33 especies de palmas, pertenecientes a 13 géneros (Geonoma, Chamaedorea, Bactris, Pholydostachys, Wettinia, Welfia, Iriartea, Socratea, Oenocarpus, Aiphanes, Astrocaryum, Attalea, Desmoncus).

Palabras clave: Estructura; Composición; Diversidad; Comunidades; Ecología; Palmas; Chocó.

\begin{abstract}
From the standpoint of importance in the composition of forests, palms are among the seven families of plants with the highest number of
\end{abstract}

1. Investigador principal, componente Ecosistémico, Instituto de Investigaciones Ambientales del Pacífico, Quibdó, Colombia. e-mail: gramirezm@unal.edu.co

2. Bióloga con Énfasis en Recursos Naturales, Universidad Tecnológica del Chocó, Quibdó, Colombia. e-mail:zulmaryvaloyes@gmail.com

3. Programa de Maestría en Ciencias Biológicas, Universidad $\mathrm{Na}-$ cional de Colombia, Instituto de Ciencias Naturales, Bogotá, Colombia.

e-mail: edledezmar@unal.edu.co

Recibido: Diciembre 15, 2007

Aceptado: Junio 24, 2008 
species in the forests of the Pacific low. Sampling relevant in the Pacific region, is found a greater number of individuals of palms by area than in any other region in the world. This study is based on a series of samples of palms in the framework of the biological expeditions of the University of Choco (2004-2005) and the study of the flora of the Pacific Research Institute of Environmental Pacific (IIAP) (2007), to analyze the diversity, distribution and composition of the appeal palm with high potential for use in the stations of the environmental IIAP in Tutunendo, Alto and San Juan High Baudó en Chocó. It was calculated the diversity alpha, beta and range, while using statistical programs for Biodiversity Pro to make the analysis of alpha diversity and measuring the rates of Shannon $H$ 'and J', Berger Paker, Simpson, Margalet, to graph and analyze the patterns of distribution and grouping of species in the community; estimate, for the analysis of the data and obtaining the percentages of those observed and estimators Chao 2, Jack Coleman and 2 and GM, and for X2 Past and the value of $P$ and to make the principal component analysis (PCA). The palms were formed in a dominant component in the vegetation of sites with a wide variety of species with high potential for local uses. These samples were found 33 species of palms, belonging to 13 genera (Geonoma, Chamaedorea, Bactris, Pholydostachys, Wettinia, Welfia, Iriartea, Socratea, Oenocarpus, Aiphanes, Astrocaryum, Attalea, Desmoncus).

Keywords: Structure; Composition; Diversity; Communities; Ecology; Palmas; Choco.

\section{INTRODUCCIÓN}

Una serie de factores físicos (humedad, altitud, temperatura y precipitación), combinados con una serie de mosaicos de hábits hacen del departamento del Chocó un sitio privilegiado en la riqueza, diversidad y distribución de las palmas. Las palmas comprenden aproximadamente 2.000 especies, agrupadas en 201 géneros, y distribuidas en las regiones tropicales y algunas subtropicales de todo el mundo. En Colombia se han encontrado 260 especies, distribuidas en 48 géneros; de estos, según Galeano \& Bernal (2004) 30 géneros y 91 especies crecen en la región del Chocó biogeográfico. Para el departamento del Chocó se registran 29 géneros y 69 especies y para la selva pluvial central 31 especies. Algunos autores afirman que a pesar de su enorme rique- za, lo que resulta más impresionante de la flora de palmas es su alto grado de endemismo. En efecto, 30 de las palmas de la región están restringidas al Pacífico colombo-ecuatoriano, o se extienden apenas hasta el centro de Panamá, pero no se encuentran en ningún otro lugar del mundo. De ellas 17 son exclusivas del Pacífico colombiano, (Bernal \& Galeano, 1993). Otros estudios informan que las tierras bajas desde el nivel del mar hasta unos 500 metros de elevación, albergan la mayor diversidad de especies de la región (Bernal \& Galeano, 1993).

Desde el punto de vista de la importancia en la composición de los bosques, las palmas se encuentran entre las siete familias de plantas con mayor número de especies en los bosques bajos del Pacífico (Gentry 1990). Además, los muestreos relevan que en la región del Pacífico, se encuentra un mayor número de individuos de palmas por área que en cualquier otra región del mundo (Gentry 1986). Con respecto a la familia se han realizado otros tipos de estudios en diferentes sitios del departamento del Chocó, como el publicado por Bernal \& Ervik (1996), quienes estudiaron la fenología de la inflorescencia y la biología floral, la visita de insectos y el transporte de polen de la palma de tagua o marfil vegetal, Phytelephas seemannii, en los bosques húmedos de la costa colombiana del Pacífico.

Para el corregimiento de Raspadura, municipio de la Unión Panamericana (Ramírez et al., 2006), mostraron a la familia Arecaceae como un componente importante del paisaje del corregimiento de Raspadura; se observó una gran variedad de géneros con alto potencial ornamental, de los cuales el género Geonoma con seis especies, fue el más representativo; otros géneros relevantes en esta investigación son: Attalea, Chamaedorea e Iriartea. Según García et al., (2002) son variados y numerosos los usos que la comunidad chocoana da a este valioso recurso entre los que se pueden enumerar: alimento, artesanías, fibra, aceites y/o grasas, palmitos, construcción, vivienda, medicina y vestido. Valois \& Pino (2004), en cuatro comunidades negras del municipio de Quibdó, encontraron que la familia Arecaceae fue la más representativa dentro las categorias de usos, con 7.2\%. Asimismo, Castro et al. (2004), en el municipio de Quibdó, corregimiento de Pacurita, reconocieron 70 morfo-especies utilizadas como alimenticias, siendo la mejor representada para este uso, Arecaceae con nueve especies y siete 


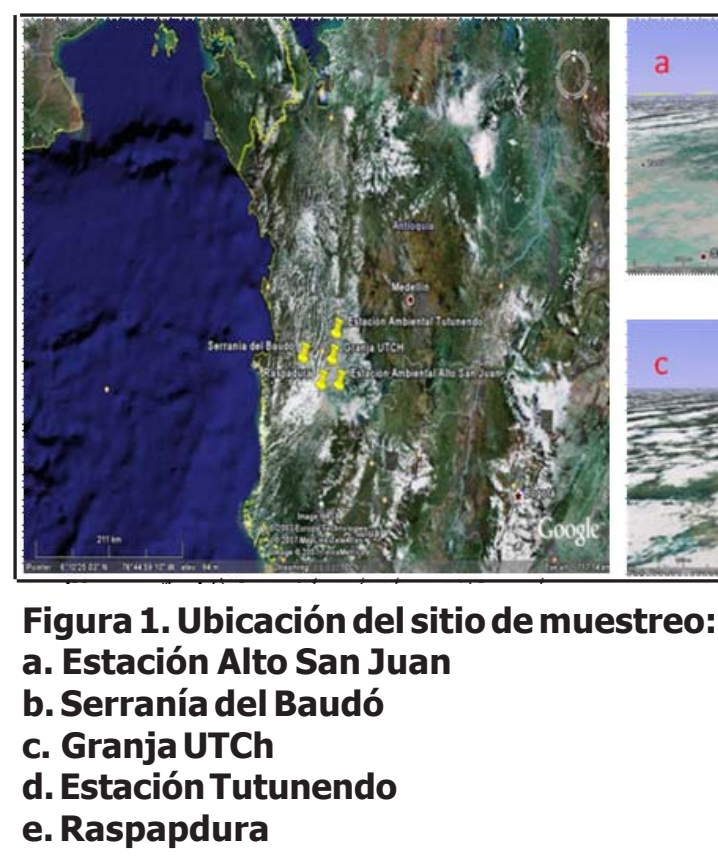

géneros equivalentes a $12.8 \%$, sobresaliendo el género Oenocarpus.

El presente estudio está basado en una serie de datos de palmas tomados de diversos trabajos realizados por Ramírez \& Ledezma, en el marco de las expediciones biológicas de la Universidad del Chocó (2004-2005) y el estudio integral de la flora del Pacífico del Instituto de Investigaciones Ambientales del Pacífico (IIAP) (2006-2007), donde el propósito era analizar la diversidad, distribución y composición del recurso palma con alto potencial de aprovechamiento en las estaciones ambientales del IIAP en Tutunendo, Alto San Juan y Alto Baudó, Chocó, además del sendero etnobiológico de Raspadura municipio de la Unión Panamericana, Chocó y en Lloró la granja de la Universidad Tecnológica del Chocó, esto, como una estrategia institucional para el conocimiento, manejo y conservación de los recursos del bosque del Chocó biogeográfico.

\section{METODOLOGÍA}

Área de estudio. El Departamento del Chocó, Colombia, está situado en la esquina noroccidental de Suramérica, entre $4^{\circ}-8^{\circ} \mathrm{N}$ y $76^{\circ}-78^{\circ} \mathrm{W}$. Es la única región de Suramérica con costa sobre dos océanos, el Atlántico al norte y el Pacífico al occidente. Tiene una extensión de $47.840 \mathrm{~km}^{2}$ y ocupa por ello el cuarto lugar entre los departamentos de Colombia. Cuenta con vías caudalosas
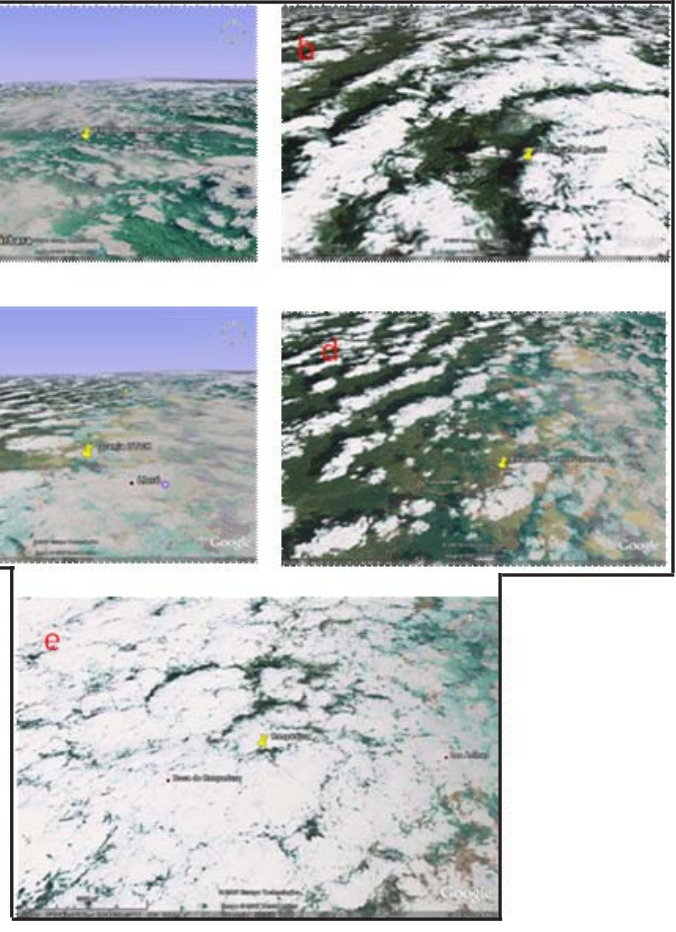

(Atrato, San Juan, Baudó, entre otras). Se pueden reconocer ocho zonas de vida (bp-PM, bmh-T, bmh-PM, bh-T, bp-MB y bp-M). Según datos registrados por la Estación Meteorológica de la Universidad Tecnológica del Chocó, en el departamento se presenta una temperatura promedio anual de $25^{\circ} \mathrm{C}$ y una humedad relativa de más de $90 \%$. E1 estudio se realizó en las estaciones ambientales del y IIAP (Alto San Juan, Alto Baudó y Tutunendo, la Granja de la UTCh y el Corregimiento de Raspadura, Chocó (Figura 1).

Método. Se utilizó el método de Gentry (1989), que consiste en establecer 10 parcelas de $80 \times 5$ $\mathrm{m}$ que corresponden a $0.4 \mathrm{~h}$ por cada localidad y colectar individuos $\geq 2 \mathrm{~cm}$, en este caso esa parte se modificó y se colectaron individuos $\geq 1 \mathrm{~cm}$. Se analizaron los datos por cada localidad estudiada no por cada transectos.

Para el análisis de la diversidad alfa, beta y gama se utilizó el método de Schluter \& Recklefs (1993). Se utilizaron los programas estadísticos de Biodiversity Pro para hacer los análisis de diversidad alfa y la medición de los índices de Shannon H'y J', Berger Paker, Simpson, Margalet, además para graficar y analizar los modelos de distribución y agrupación de las especies en la comunidad; EstimateS, para el análisis de los datos y la obtención de los porcentajes de los 
Comunidades de palmas en cinco localidad del Chocó. G Ramírez, et al.

Tabla 1

Matriz de datos de las cinco comunidades de palmas del Chocó

\begin{tabular}{|c|c|c|c|c|c|}
\hline 33 & 6 & & & & \\
\hline 146 & 1000 & 60 & 56 & 67 & 1329 \\
\hline 72 & 300 & 92 & 23 & 54 & 541 \\
\hline 1 & 56 & 0 & 50 & 43 & 150 \\
\hline 1 & 56 & 26 & 30 & 23 & 136 \\
\hline 0 & 54 & 0 & 23 & 56 & 133 \\
\hline 12 & 0 & 0 & 77 & 32 & 121 \\
\hline 18 & 45 & 0 & 12 & 45 & 120 \\
\hline 5 & 39 & 18 & 30 & 12 & 104 \\
\hline 46 & 43 & 7 & 0 & 0 & 96 \\
\hline 10 & 67 & 19 & 0 & 0 & 96 \\
\hline 54 & 34 & 1 & 0 & 0 & 89 \\
\hline 8 & 0 & 10 & 15 & 19 & 52 \\
\hline 27 & 15 & 0 & 6 & 0 & 48 \\
\hline 12 & 10 & 0 & 0 & 24 & 46 \\
\hline 4 & 27 & 0 & 0 & 12 & 43 \\
\hline 1 & 15 & 1 & 4 & 21 & 42 \\
\hline 5 & 26 & 9 & 0 & 0 & 40 \\
\hline 0 & 8 & 25 & 0 & 0 & 33 \\
\hline 0 & 0 & 30 & 0 & 0 & 30 \\
\hline 1 & 11 & 0 & 0 & 13 & 25 \\
\hline 0 & 0 & 23 & 0 & 0 & 23 \\
\hline 0 & 0 & 0 & 21 & 0 & 21 \\
\hline 0 & 0 & 20 & 0 & 0 & 20 \\
\hline 6 & 0 & 9 & 0 & 0 & 15 \\
\hline 0 & 0 & 11 & 0 & 0 & 11 \\
\hline 0 & 10 & 0 & 0 & 0 & 10 \\
\hline 0 & 8 & 1 & 0 & 0 & 9 \\
\hline 0 & 7 & 1 & 0 & 0 & 8 \\
\hline 0 & 0 & 4 & 0 & 4 & 8 \\
\hline 0 & 0 & 3 & 0 & 0 & 3 \\
\hline 1 & 1 & 0 & 0 & 1 & 3 \\
\hline 0 & 0 & 2 & 0 & 0 & 2 \\
\hline 0 & 0 & 1 & 0 & 0 & 1 \\
\hline
\end{tabular}

observados y los estimadores de Chao 2, Jack 2 y Coleman y MM; y Past para obtener $\mathrm{X}^{2}$ y el valor de $P$ y para hacer el análisis de componentes principales (PCA).

\section{RESULTADOSYDISCUSIÓN}

Las palmas se constituyeron en un componen- te dominante en la vegetación de los sitios muestreados; tiene una gran variedad de especies con alto potencial para usos locales. En estos muestreos se encontraron 33 especies de palmas, pertenecientes a 13 géneros (Geonoma, Chamaedorea, Bactris, Pholydostachys, Wettinia, Welfia, Iriartea, Socratea, Oenocarpus, Aiphanes, Astrocaryum, Attalea, Desmoncus) de los cuales los géneros Geonoma, Chamaedoreay Bactris fueron los más representativos en casi todos los sitios de muestreo. Otras observaciones interesantes las constituyen la alta abundancia y grandes poblaciones de la asociación entre Wettinia quinaria y Welfia regia (Tabla 1 ).

Análisis de la diversidad alfa. Este análisis se basó en los índices de Shannon H'y J', para analizar riqueza y equidad de especies; Simpson, para analizar la dominancia; Margalef, para analizar diversidad y abundancia; Berger Parker, para analizar la abundancia y dominancia.

Según el índice de Shannon $\mathrm{H}^{\prime}$ la menor variación se observó en la localidad 2 (0.772) y una tendencia en las demás de poca variabilidad. El Shannon J muestra la distribución de la equidad más baja para la localidad $2(0.575)$.

Según Simpson la mayor dominancia se expresa en la localidad 2 (0.329).

- Margalef muestra una baja diversidad en la localidad 2 (9.803) pero esta difiere en relación con el total (9.059) puede deberse a que el total es la suma de todas las localidades las cuales no aportan muchas especies nuevas a la diversidad del total.

Según Berger-Parker la localidad que más contribuye a la abundancia es la 2 con 1.838 , teniendo en cuenta que en esta se encuentra la especie más dominante con cerca de 1.000 individuos.

Utilizando el método de Schluter \& Recklefs (1993), para calcular diversidad alfa, beta y gama, se encontró que una diversidad alfa promedio $=18$; diversidad beta promedio $=0.37$ y la diversidad gama $=33$. Al analizar estos resultados y teniendo en cuenta que la diversidad gama es el producto de $\alpha^{*} \beta^{*} \mathrm{~N}$ localidades $=18^{*} 0.37^{*} 5=33$, se observan en estos resultados que el mayor aporte a la diversidad regional lo hace la diversidad local porque el valor del recambio es muy bajo y por ende no aporta mucho al aumento de la diversidad regional.

Curvas de acumulación de especies. Aquí se graficaron y analizaron las especies de palmas 
Bioetnia Volumen 5 No 1 (enero-junio), 2008

Tabla 2

Distribución de las abundancias de especies de palmas por localidades

\begin{tabular}{|c|c|c|c|c|c|c|c|c|c|c|c|c|c|}
\hline \multicolumn{3}{|c|}{ Sample } & \multicolumn{2}{|c|}{ Sample } & \multicolumn{3}{|c|}{ Sample } & \multicolumn{3}{|c|}{ Sample } & \multicolumn{3}{|c|}{ Sample } \\
\hline 1 & & 1 & 2 & 2 & 3 & & 3 & 4 & & 4 & 5 & & 5 \\
\hline Species & 1 & & Species 1 & 1000 & Species & 2 & 92 & Species & & 77 & Species & 1 & 67 \\
\hline Species & 2 & & Species 2 & 300 & Species & 1 & 60 & Species & 1 & 56 & Species & 5 & 56 \\
\hline Species & 11 & 54 & Species 10 & 67 & Species & 19 & 30 & Species & 3 & 50 & Species & 2 & 54 \\
\hline Species & 9 & 46 & Species 3 & 56 & Species & 4 & 26 & Species & 4 & 30 & Species & 7 & 45 \\
\hline Species & 13 & & Species 4 & 56 & Species & 18 & 25 & Species & 8 & 30 & Species & 3 & 43 \\
\hline Species & 7 & & Species 5 & 54 & Species & 21 & 23 & Species & 2 & 23 & Species & 6 & 32 \\
\hline Species & 6 & 12 & Species 7 & 45 & Species & 23 & 20 & Species & 5 & 23 & Species & 14 & 24 \\
\hline Species & 14 & 12 & Species 9 & 43 & Species & 10 & 19 & Species & 22 & 21 & Species & 4 & 23 \\
\hline Species & 10 & & Species 8 & 39 & Species & 8 & 18 & Species & 12 & 15 & Species & 16 & 21 \\
\hline Species & 12 & & Species 11 & 34 & Species & 25 & 11 & Species & 7 & 12 & Species & 12 & 19 \\
\hline Species & 24 & 6 & Species 15 & 27 & Species & 12 & 10 & Species & 13 & 6 & Species & 20 & 13 \\
\hline Species & 8 & 5 & Species 17 & 26 & Species & 17 & 9 & Species & 16 & 4 & Species & 8 & 12 \\
\hline Species & 17 & & Species 13 & 15 & Species & 24 & 9 & Species & 9 & 0 & Species & 15 & 12 \\
\hline Species & 15 & & Species 16 & 15 & Species & 9 & 7 & Species & 10 & 0 & Species & 29 & 4 \\
\hline Species & 3 & 1 & Species 20 & 11 & Species & 29 & 4 & Species & 11 & 0 & Species & 31 & 1 \\
\hline Species & 4 & 1 & Species 14 & 10 & Species & 30 & 3 & Species & 14 & 0 & Species & 9 & 0 \\
\hline Species & 16 & & Species 26 & 10 & Species & 32 & 2 & Species & 15 & 0 & Species & 10 & 0 \\
\hline Species & 20 & & Species 18 & 8 & Species & 11 & 1 & Species & 17 & 0 & Species & 11 & 0 \\
\hline Species & 31 & 1 & Species 27 & 8 & Species & 16 & 1 & Species & 18 & 0 & Species & 13 & 0 \\
\hline Species & 5 & 0 & Species 28 & 7 & Species & 27 & 1 & Species & 19 & 0 & Species & 17 & 0 \\
\hline Species & 18 & & Species 31 & 1 & Species & 28 & 1 & Species & 20 & 0 & Species & 18 & 0 \\
\hline Species & 19 & & Species 6 & 0 & Species & 33 & 1 & Species & 21 & 0 & Species & 19 & 0 \\
\hline
\end{tabular}

sp13 con 2 individuos en la localidad tres, en la misma localidad Geonoma sp11 con tres individuos, Geonoma sp12 con 11 individuos, Geonoma sp 8 con 20 individuos, Astrocarium standleyanum con 23 individuos y Euterpe $s p 1$ con 30 individuos que hacen de esta localidad la más importante en recambio de especies por el aporte de las especies raras. Esto muestra una respuesta biológica al aumento en el gradiente altitudinal, porque este muestreo se hizo en una subregión diferente a las anteriores; aunque en este trabajo no se tuvieron en cuenta variables ambientales, el dominio y aparición de ciertos grupos de palmas como las de la Subtribu Geonomea, hace pensar que hay un incremento moderado en las variables ambientales que coincide con el aumento notable de palmas que no se habían colectado en las otras localidades, lo que también puede estar mostrando el estado de conservación de la locali- dad, porque el grupo antes mencionado tiene uno requerimientos bastante particulares, por ser plantas de sotobosque, como un alto porcentaje de cobertura del dosel, sumado una gran cantidad de nutrientes en el suelo y una fuerte interacción con la fauna silvestre para la polinización y dispersión de sus semillas.

El patrón global de la distribución de especies se ve influido por la abundancia de 2 especies Wettinia quinaria con (1.329 individuos) y Welfia regia con (541 individuos) que sumadas corresponden a $54.8 \%$ de la distribución general de la comunidad de palmas, seguido se encuentran muchas especies con abundancia intermedia que van desde 150 individuos hasta 89. En ese mismo orden abundancias de que van desde 52 hasta 20 y por último las especies raras con menos de 15 individuos.

Se realizaron varias pruebas para determinar 
el modelo que mejor se ajusta a los datos obtenidos. Según el método planteado por Magurran (1998), se examinaron los modelos de Log normal, Serie Logaritmica y palo quebrada, utilizando como criterio de bondad de ajuste la prueba $\mathrm{chi}^{2}$. El valor de $X^{2}=16.59$ para $g l=9$ se obtuvo un $p=0.0609$ como la probabilidad es mayor que el nivel de significancia fijado $(\mathrm{p}<0.05)$, no se puede rechazar $\mathrm{H}_{\mathrm{o}}$, por tanto con los datos obtenidos en este estudio se puede concluir estadísticamente que concuerdan con el modelo de abundancia de especie log normal.

Algo importante para comentar es la distribución general de las especies las cuales en su mayoría (30 especies) se encuentran en forma agrupada $(90.9 \%)$ y el resto $3(9.1 \%)$ tienen una distribución aleatoria.

Análisis de componentes principales. La gráfica describe las variables (riqueza y abundancia) y la distribución de los sitios de acuerdo con las mismas. Tabla 1 indica que la mayor cantidad de variación en los datos está explicada por el primer componente (autovalor y porcentaje de varianza más altos). En las otras tablas se muestran las cargas de cada variable dentro de cada componente, es decir, las variables que más están aportando al primer componente. En este caso la variación está dada por las altas abundancias de Wettinia quinaria y Welfia regia, mientras que Euterpe precatoria y Bactris maraja son las que más aportan en la variación de los datos para el componente 2 .

Se observan tres grupos claramente definidos a partir de la utilización de la variable abundancia; además, se ve que el componente 1 explica 97\% de los resultados del análisis y que la conformación de los grupos se debe a la similaridad de la abundancia de las especies compartidas por los grupos. Bajo estas condiciones se forman los siguientes grupos (Figura 1):

- Tutunendo

- Raspadura-Lloró

- Baudó-San Juan

Añadiendo más sobre los pesos de la variable, se observa que la especie 1 es la que influye la distribución de los valores y la conformación de los grupos; de esta misma forma se nota una gran similitud de los resultados de este análisis con la distribución de las abundancias que se observó en pasos anteriores. La explicación también se puede deber a que esta especie se presenta en casi todo los muestreos y a la combinación entre pocas especies muy raras con baja abundancia y varias especies con abundancias muy altas, que son las que están determinando la agrupación de los sitios a través de los pesos de las especies por sus abundancias (Tabla 3 ).

Diversidad vs. variables de agua (precipitación y dias húmedos por año). Haciendo una observación detallada de la zona y después de analizar su estructura paisajística se determinó que la diversidad local es muy alta; esto puede inferir de la correlación positiva que existe entre las altas precipitaciones de la zona $(9.000 \mathrm{~mm}$ anuales y cerca de 280 días húmedos por año) y la gran diversidad de palmas, análisis que corrobora lo planteado por Bjorholm et al. (2005), que aducen la alta diversidad de palmas en el Chocó se presenta por la altas precipitaciones y por la cantidad de días húmedos en el año.

Diversidad vs. lactitud y altitud. Además, a esto se suma que los muestreos se realizaron a los $05^{\circ} \mathrm{N}$, esto también comprueba las afirmaciones de Bjorholm et al. (2005), quienes proponen en su estudio que además de las variables de agua (precipitación y días húmedos por año) la latitud también es muy influyente en la distribución de la alta diversidad de palmas en el Chocó porque el rango se encuentra entre los $7^{\circ} \mathrm{N}$ y $7^{\circ} \mathrm{S}$ e inicia la disminución de esta diversidad que a partir de los $10^{\circ} \mathrm{N}$ y $10^{\circ} \mathrm{S}$. El presente estudio muestra también la relación existente entre la altitud y la diversidad de palmas, pues los muestreos se hicieron en un rango de 103 a $112 \mathrm{msnm}$ que es una cualidad que según Gentry (1993), está directamente relacionada con la alta diversidad de palmas en las tierras bajas del Pacífico.

\section{AGRADECIMIENTOS}

A Dios todo poderoso, al director del Instituto de Investigaciones Ambientales del Pacífico, Dr. Willian Klinger, al director del Instituto de Ciencias Naturales de la Universidad Nacional de Bogotá, Dr. Jaime Aguirre, a mis directores de tesis Gloria Galeano y Rodrigo Bernal, a la coordinadora de la investigación bióloga Angélica Asprilla, a los integrantes de los grupos de investigaciones de Biosistemática y Palmas Neotropicales, al camarógrafo Tomás Salas, al grupo de trabajadores sociales y a mis colegas Jhon Jairo Cuesta, Leider Palacios, Yairton Cuesta y Enrique Renteria. 
Bioetnia Volumen 5 № 1 (enero-junio), 2008

Tabla 3

Valores de los componentes más representativos de la comunidad de palmas del Chocó

\begin{tabular}{|c|c|c|c|}
\hline \multicolumn{2}{|c|}{ Componente 1} & \multicolumn{2}{|c|}{ Componente 2} \\
\hline Variable & Carga & Variable & Carga \\
\hline Wettiniaquinaria & 0,9609 & Euterpeprecatoria & $-0,4904$ \\
\hline Welfiaregia & 0,2514 & Bactrismaraja & $-0,4591$ \\
\hline Socretea & 0,06258 & Oenocarpussp1 & $-0,3653$ \\
\hline Iriarthea & 0,03503 & Welfiaregia & 0,3371 \\
\hline Geonomasp1 & 0,03485 & Geonomasp2 & 0,2189 \\
\hline Euterpeprecatoria & $-0,0348$ & Geonomasp1 & 0,2136 \\
\hline Oenocarpussp1 & 0,03332 & Oenocarpussp2 & $-0,1826$ \\
\hline Bactrismaraja & 0,03051 & Euterpe & 0,1648 \\
\hline Oenocarpussp2 & 0,02737 & Geonomasp9 & 0,1336 \\
\hline Geonomasp2 & 0,02581 & Iriarthea & $-0,1308$ \\
\hline Geonomasp3 & 0,024 & Astrocaryum & 0,1264 \\
\hline Pholidostachyssp 1 & 0,02364 & Socretea & 0,1151 \\
\hline Bactris & 0,022 & Geonomasp7 & $-0,1142$ \\
\hline Cotoblastus & $-0,01411$ & Geonomasp8 & 0,1099 \\
\hline Pholidostachyssp2 & 0,01039 & Bactris & $-0,1075$ \\
\hline Geonomasp4 & 0,009111 & Attalea & $-0,0996$ \\
\hline Euterpe & $-0,008371$ & Wettiniaquinaria & $-0,08568$ \\
\hline Geonomasp10 & 0,00803 & Geonomasp4 & 0,07503 \\
\hline Attalea & 0,008021 & Chamaedoreasp2 & 0,07496 \\
\hline Geonomasp5 & 0,007619 & Cotoblastus & $-0,06544$ \\
\hline Chamaedoreasp1 & 0,006991 & Geonomasp12 & 0,06044 \\
\hline Geonomasp7 & $-0,006491$ & Pholidostachyssp 1 & 0,05836 \\
\hline Astrocaryum & $-0,006418$ & Geonomasp5 & $-0,05079$ \\
\hline Geonomasp8 & $-0,00558$ & Geonomasp6 & $-0,04571$ \\
\hline Chamaedoreasp2 & $-0,003537$ & Geonomasp3 & $-0,0418$ \\
\hline Geonomasp12 & $-0,003069$ & Geonomasp11 & 0,01648 \\
\hline Desmoncussp1 & $-0,002235$ & Geonomasp13 & 0,01099 \\
\hline Geonomasp6 & 0,001624 & Desmoncussp1 & 0,00665 \\
\hline Geonomasp9 & 0,001333 & Chamaedoreasp3 & 0,005495 \\
\hline Geonomasp11 & $-0,0008371$ & Pholidostachyssp2 & $-0,004747$ \\
\hline Aiphanes acaulis & 0,0005881 & Chamaedoreasp1 & 0,002172 \\
\hline Geonomasp13 & $-0,000558$ & Geonomasp10 & 0,001697 \\
\hline Chamaedoreasp3 & $-0,000279$ & Aiphanes acaulis & $-5.57 E-02$ \\
\hline
\end{tabular}

\section{LITERATURA CITADA}

Bernal, R. y G. G. Galeano. 1993. Palmas del andén Pacífico. En: Leyva, P. (ed). Colombia Pacífico. Tomo I. Bogotá: Editorial del Fondo FEN, Instituto de Ciencias Naturales, Universidad Nacional de Colombia. p. 220-31.

Bernal, R. G. y F. Ervik. 1996. Floral biology and pollination of the dioecious palm Phytelephas seemannii in Colombia: an adaptation to staphylinid beetles. Biotropica. 28: 682-96.

Biodiversty Profesional. 1997. Version 2.

Beck, H. 2006. A review of peccary-palm interactions and their ecological ramifications across the neotropical. J Mammal. 87:519-30.

Bjorholm, S., J-C, Svenning, F. Skov. y H. Balslev. 2005. Environmental and spatial controls of palm (Arecaceae) 


\section{Comunidades de palmas en cinco localidad del Chocó. G Ramírez, et al.}

species richness across the Americas. Global Ecol Biogeograp. 14:423-9.

Bonilla, M. A. 2003. Análisis de la estructura de una comunidad biológica. En: Notas de clases prácticas de ecología. Bonilla, M. A. y G. Guillot (Editores). Bogotá: Facultad de Ciencias, Universidad Nacional de Colombia.

Castro, A., D. Abadía y N. Pino. 2004. Plantas silvestres alimenticias de uso tradicional en las comunidades de Pacurita, San José de Purré y Guayabal, municipio de Quibdó, Chocó. Revista Institucional Universidad Tecnológica del Chocó.18: 37-42.

Collwell, R. 2006. Statistical estimation of richness and shared species for samples. Version 800. University of Connecticut, USA.

Galeano, G. y R. Bernal. 2004. Catálogo de espermatofitos en el Chocó Biogeográfico familia Arecaceae. En: Rangel-Ch, J.O. (Editor). Colombia diversidad biótica IV: El Chocó biogeográfico/ Costa Pacífica. Bogotá: Instituto de Ciencias Naturales, Universidad Nacional de Colombia. p. 135-48.

Gentry, A. H. 1993. Riqueza de especies del Pacífico. En: Leyva, P. (ed). Colombia Pacífico. Tomo I. Bogotá: Editorial del Fondo
FEN, Instituto de Ciencias Naturales, Universidad Nacional de Colombia. p. 201-19.

Hammer, O. Haper, D. A. Ty Ryan, P. D. 2001. PAST: Palaentological Stotatistics Sofware Package for Education and Data Analyzis. Palaentol Electr. 4 (1): 9.

Magurran, A. 1989. Lecciones e interpretación de las medidas de diversidad. En: Diversidad ecológica y su medición. Barcelona: Ediciones Vedra.

Moreno, C. E. 2001. Métodos para medir la biodiversidad. Vol. 1. Zaragoza: M\&T-Manuales y Tesis SEA. 84 pp.

Ramirez, G., E. Ledezma, y N. Pino. 2006. The most important palm genera with ornamental potential of the region Raspadura, city of Unión Panamericana-Chocó, Colombia. Lyonia J Ecol Applic. 10 (2): 47-63.

Ricklefs, R, Ey Schulter, D. 1993. Species diversity an introduction to the problem. Species diversity in ecological communities "Historical and geographical perspectives". Chicago: Universidad de Chicago. p. 1-11,

Valois, H. \& N. Pino. 2004. Ethnobotany of four black communities of the municipality of Quibdó, Chocó-Colombia. Lyonia JEcol Applic. 7 (2): 61-9. 